\title{
Structure of $\mathrm{C} 3 \mathrm{~b}$-factor $\mathrm{H}$ and implications for host protection by complement regulators
}

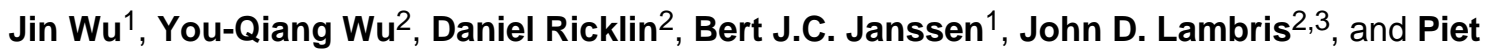 \\ $\operatorname{Gros}^{1,3}$
}

${ }^{1}$ Crystal and Structural Chemistry, Bijvoet Center for Biomolecular Research, Department of Chemistry, Faculty of Science, Utrecht University, Padualaan 8, $3584 \mathrm{CH}$ Utrecht, The Netherlands ${ }^{2}$ Department of Pathology \& Laboratory Medicine, University of Pennsylvania, 401 Stellar Chance, Philadelphia, PA 19104, USA

\begin{abstract}
Factor $\mathrm{H}(\mathrm{FH})$ is an abundant regulator of complement activation and protects host cells from selfattack by complement. Here we provide insights into the regulatory activity of FH by solving the crystal structure of the first four domains of $\mathrm{FH}$ in complex with its target $\mathrm{C} 3 \mathrm{~b}$. FH interacts with multiple domains of $\mathrm{C} 3 \mathrm{~b}$, covering a large, extended surface area. The structure indicated that $\mathrm{FH}$ destabilizes the $\mathrm{C} 3$ convertase by competition and electrostatic repulsion and that FH enables proteolytic degradation of $\mathrm{C} 3 \mathrm{~b}$ by providing a binding platform for the protease factor $\mathrm{I}$, while stabilizing the overall domain arrangement of $\mathrm{C} 3 \mathrm{~b}$. The results offer general models for complement regulation and provide structural explanations for disease-related mutations in both $\mathrm{FH}$ and $\mathrm{C} 3 \mathrm{~b}$.
\end{abstract}

\section{Keywords}

Complement system; Innate immunity; Regulator of complement activation; Factor H;

Complement component $\mathrm{C} 3 \mathrm{~b}$

The efficacy of the complement-mediated immune response relies on a delicate balance between activation and regulation. While the continuous generation of the strong opsonin complement component $3 \mathrm{~b}(\mathrm{C} 3 \mathrm{~b})$ by the alternative pathway (AP) allows a rapid reaction to foreign or abnormal cells, its indiscriminate deposition may potentially cause host tissue

\footnotetext{
Users may view, print, copy, and download text and data-mine the content in such documents, for the purposes of academic research, subject always to the full Conditions of use:http://www.nature.com/authors/editorial_policies/license.html\#terms

Correspondence Coordinates and structure factors of the $\mathrm{C} 3 \mathrm{~b}-\mathrm{FH}(1-4)$ complex have been deposited in the Protein Data Bank with succession number 2WII. Correspondence and requests for materials should be addressed to P.G. (HUp.gros@uu.nlUH) or J.D.L. (Lambris@upenn.edu).

${ }^{3}$ Shared senior authorship

Author contributions

J.W. purified C3 and C3b. Y-Q.W. expressed and purified FH(1-4) and performed cofactor assays. D.R. performed decay acceleration, antibody competition, and direct binding studies. J.W. crystallized the complex, collected data, determined, refined and analyzed the structure. B.J.C.J. helped with all stages of structure determination and analysis. J.D.L. and P.G. conceived and supervised the project. J.W., D.R., and P.G. wrote the manuscript.

Competing interests The authors declare no financial interests
} 
damage. Host cells are therefore protected by proteins of the regulator of complement activation (RCA) family, which either impair the generation of new $\mathrm{C} 3 \mathrm{~b}$ by accelerating the decay of the $\mathrm{C} 3$ convertases or act as cofactor for factor I (FI) in degrading existing C3b1,2. In addition to cell-surface bound RCA like decay accelerating factor (DAF, also known as CD55 [http://www.signaling-gateway.org/molecule/query?afcsid=A000571]), membrane cofactor protein (MCP or CD46 [http://www.signaling-gateway.org/molecule/query? afcsid=A000568]) and complement receptor 1 (CR1 or CD35 [http://www.signalinggateway.org/molecule/query?afcsid=A000541]), the soluble and highly abundant regulator factor $\mathrm{H}(\mathrm{FH})$ offers an additional layer of protection as it controls the steady-state AP activation in circulation. Furthermore, FH may be recruited to host membranes by recognizing and binding self components, such as glycosaminoglycans, and thereby prevent opsonization of host tissue with low expression of surface RCA1,3.

The importance of FH in maintaining a well-balanced immune response is reflected by the increasing number of diseases with strong association to mutations and polymorphisms in the $\mathrm{CFH}$ gene, as seen in age-related macular degeneration (AMD), atypical hemolytic uremic syndrome (aHUS), and membranoproliferative glomerulonephritis type II (MPGNII)4,5. Several pathogenic microorganisms evade attack of the complement cascade by expressing structural homologues of FH or recruiting host FH to their surfaces via FHbinding proteins6,7. Owing to their vital role in immune modulation, therapeutic targeting of FH as well as other RCA proteins is considered important for the treatment of diseases associated with abnormal or loss of complement control8,9.

FH is formed by a linear string of complement-control-protein (CCP) domains that consist of about 60 residues and that are common to all RCA proteins1. The first four domains (CCP1-4) of FH are necessary and sufficient for regulation of complement in the fluid phase, whereas host cell specificity is determined by domains CCP5-20 of FH1,10,11. Despite a wealth of functional and structural data on CCP domains of various complement regulators, our understanding of the two molecular mechanisms that protect host cells, referred to as 'decay-acceleration activity' and 'cofactor activity', still remains limited.

Here we provide insights into the mechanisms of host protection against complement activation by presenting the crystal structure of the four functional domains of FH (CCP1-4) in complex with C3b. By combining our structural model with biochemical and biophysical data as well as with findings from previous mutational studies, we are able to dissect individual processes and correlate disease-related mutations with functional consequences. Furthermore, our findings allow us to extrapolate to other members of the RCA family and develop a more general model for complement regulation.

\section{RESULTS}

\section{Crystal structure of the C3b-FH(1-4) complex}

For this study, we expressed the N-terminal four CCP domains (amino acid residues 1-246) of FH, hereafter called $\mathrm{FH}(1-4)$, which are known to mediate both of the regulatory activities of FH10 (Fig. 1a). This recombinant FH(1-4) bound C3b with an affinity of $\mathrm{K}_{\mathrm{D}}=$ 
$11 \mu \mathrm{M}$ (Fig. 1b,c) and was functionally active in vitro with respect to both decayacceleration and cofactor activity (Fig 1d,e).

We crystallized the complex of C3b-FH(1-4) and determined the structure to 2.7- $\AA$ resolution (Fig. 2 and Table 1). The structure revealed a large and discontinuous interface between $\mathrm{FH}(1-4)$ and $\mathrm{C} 3 \mathrm{~b}$ that stretches over a distance of $100 \AA$ and buries a surface area of $\sim 4,500 \AA 2$ (Fig. 2a). Despite the putative flexibility between individual CCP domains of $\mathrm{FH}, \mathrm{FH}(1-4)$ bound to $\mathrm{C} 3 \mathrm{~b}$ shows only small differences in domain orientations when compared to the solution structures of CCP1-2 and CCP2-3 (ref. 12). Tilt angle between CCP1 and CCP2 altered by $\sim 10^{\circ}$, which led to closer proximity of CCP1 towards C3b (Fig. $2 \mathrm{~b}$ and Supplementary Table 1 online). A kink occurred between CCP 3 and CCP4 and was responsible for an overall L-shape appearance of $\mathrm{FH}(1-4)$, which is consistent with smallangle X-ray scattering data of FH CCP1-5 (ref. 13). The structure of C3b revealed its typical arrangement of 12 domains formed by the $\beta$ chain (amino acid residues 1-645) and the $a$ ' chain (amino acid residues 727-1,641)14,15, obtained after proteolytic activation of the native $\mathrm{C} 3$ (Fig. 2). The core of the structure is formed by eight macroglobulin (MG) domains and a linker (LNK) domain. Inserted between MG7 and MG8 are a 'complement C1r/C1s, UEGF, BMP1' (CUB) domain and a thioester containing domain (TED), which is responsible for covalent attachment to target surfaces. The $\mathrm{C}$-terminus is extended by a C345C domain, which is common to complement components C3, C4 and C5. Two differences in domain orientations were observed between $\mathrm{C} 3 \mathrm{~b}-\mathrm{FH}(1-4)$ and unbound $\mathrm{C} 3 \mathrm{~b}$ : the C-terminal $\mathrm{C} 345 \mathrm{C}$ domain repositions, which we attribute to crystal packing, and the CUB and TED domains rotate by $\sim 12^{\circ}$ (Fig. 2 b and Supplementary Fig 1, Supplementary Fig 2 online) 14,15. The change in CUB and TED was likely due to interactions with $\mathrm{FH}(1-$ 4); however, variations in CUB-TED positionings have been observed among other structures of C3b (Fig. 2b and Supplementary Figure 1 online)14,15.

The C3b-FH(1-4) interface consisted of four contact regions spanning the length of $\mathrm{FH}(1-4)$ (Fig. 3 and Supplementary Table 2 online). The bottom half of CCP1 and the CCP1-CCP2 linker attached through hydrophobic interactions and salt bridges to the acidic a' N-terminal (a'NT) region (amino acid residues 727-746) and the MG7 domain in C3b. Antibody binding to MG7-MG8 inhibited the interaction of $\mathrm{C} 3 \mathrm{~b}$ with both $\mathrm{FH}(1-4)$ and full-length $\mathrm{FH}$ (Supplementary Fig. 3 online)16, which confirms the a'NT and MG7 region as a primary binding site for FH17,18. The second major binding site involves a patch of conserved hydrophobic residues surrounded by hydrophilic residues on CCP2 that interacted with MG6 of C3b (Fig. 2a and Supplementary Fig. 4,Supplementary Fig. 5 online). At the third site, $\mathrm{CCP} 3$ contacted residues of both the $a^{\prime}$ and the $\beta$ chain of $\mathrm{C} 3 \mathrm{~b}$ and bridged MG 2 and CUB, which harbors the three scissile bonds that are cleaved by FI. Interestingly, the hypervariable loop of FH CCP3 (amino acid residues 139-145) that was disordered in the previous NMR structure 12, was ordered in the C3b-FH(1-4) complex and interacted with the CUB domain of C3b. At the fourth and final site, CCP4 formed another bridge between MG1 and TED, which was confirmed by blocking experiments using a TED-specific antibody (Supplementary Fig. 3 online). The arrangement of the a'NT, MG7, CUB and TED domains are known to substantially change during the conversion of $\mathrm{C} 3$ to $\mathrm{C} 3 \mathrm{~b} 14,15,19,20$, which clearly explains the specificity of $\mathrm{FH}$ for $\mathrm{C} 3 \mathrm{~b}$ over $\mathrm{C} 3$. In summary, the complex revealed an 
extensive interface that involves multiple domains of $\mathrm{C} 3 \mathrm{~b}$ and all domains $\mathrm{FH}(1-4)$, which is supported by binding studies in this work (Fig. 1b-c, Supplementary Fig.4 online) and previous reports16,17,18.

\section{Structural basis of decay-acceleration activity}

The ability of $\mathrm{FH}(1-4)$ to dissociate the protease fragment $(\mathrm{Bb})$ from the alternative pathway $\mathrm{C} 3$ convertase complex (C3bBb; Fig. 1d) suggests competition for the same binding region on $\mathrm{C} 3 \mathrm{~b}$. We therefore compared the $\mathrm{C} 3 \mathrm{~b}-\mathrm{FH}(1-4)$ structure with that of the convertase, stabilized by the staphylococcal inhibitor SCIN (C3bBbSCIN; Fig. 4a)21, by superimposing the $\beta$ chain of the $\mathrm{C} 3 \mathrm{~b}$ molecules from both complexes. The superposition showed a large steric clash between CCP1-2 of FH(1-4) and the $\mathrm{Bb}$ fragment of the convertase. The Nlinked glycan at Asn-260 in Bb contributed to the observed clash (Supplementary Fig. 6 online). Accordingly, deletion of this glycan by mutation N260D renders the convertase less sensitive to decay acceleration22. In addition to steric hindrance, the complementary surfaces of $\mathrm{FH}$ and $\mathrm{Bb}$ are both negatively charged, which implied that electrostatic repulsion contributed to destabilization of the $\mathrm{C} 3 \mathrm{bBb}$ complex (Fig. 4b). These results revealed a functional role for $\mathrm{CCP} 1-2$ in displacing $\mathrm{Bb}$ and a supportive role of CCP3-4 in binding to $\mathrm{C} 3 \mathrm{~b}$.

In order to propose a general model of decay acceleration, we compared our data with the structure and mutagenesis data of DAF23,24. The overlay of the functional CCP2-3 domains of DAF onto FH CCP1-2 showed that DAF exhibited similar residues at the C3b contact sites (Supplementary Fig. 5 online). Moreover, residues critical for DAF activity, which are located in the CCP2-3 linker and on the CCP3 surface, faced $\mathrm{Bb}$ in the $\mathrm{C} 3 \mathrm{bBb}$ complex (Fig. 4c)24. Similar to FH, the proposed binding mode of DAF resulted in a steric overlap with $\mathrm{Bb}$. However, the critical residues on DAF facing $\mathrm{Bb}$ in the $\mathrm{C} 3 \mathrm{bBb}$ complex were predominantly hydrophobic and not negatively charged as in the case of FH (Fig. 4b,c and Supplementary Fig. 5 online). The hydrophobic patch on DAF faced the $\beta$ F-a 7 loop of the von Willebrand factor A (VWA) domain in Bb (Fig. 4a,c). This section of the VWA domain was hidden in the pro-enzyme FB, which possibly explains why DAF binds Bb but not fulllength FB25. Putatively, interaction of DAF with this loop may induce a low affinity state of the metal-ion dependent adhesion site (MIDAS) in $\mathrm{Bb}$ and thereby disrupt the $\mathrm{C} 3 \mathrm{bBb}$ complex, similar to the allostery observed in integrin I domains 26 . However, the structural observations do not readily explain the effects of mutations at Lys-298 and Tyr-338 in FB on the decay-acceleration activity of DAF, CR1 and FH22,27. Possibly, these mutations in the VWA domain, distal from the RCA interaction side, may affect the allosteric mechanism.

\section{Implications for cofactor activity}

FH supports two, out of three possible, cleavages in the CUB domain of C3b by FI (between R1281-S1282 and R1298-S1299) that yield the inactive iC3b species (Fig. 1e)28. The crystal structure revealed that FH CCP2-3 bound to C3b adjacent to the CUB domain (Fig. 2a, Fig. 5a) with the hypervariable loop of CCP3 directly contacting CUB (Fig. 5a,b)10. Cleavage site R1281-S1282 was well-exposed, whereas R1298-S1299 was occluded in the complex (Fig. 5b), which confirms the notion that R1281-S1282 is cleaved first and suggests 
that cleavage of R1298-S1299 requires conformational changes. The strong influence of ionicity for the binding of FI indicates that polar interactions are highly important for this interaction29. FH CCP1-3 indeed exhibited several conserved and charged patches on its surface (Fig. 5a and Supplementary Fig. 7 online), which may be involved in the binding of FI. For the FH-homologue vaccinia virus complement control protein (VCP), CCP2 was shown to have a dominant role in complement inhibition. Mutation of four residues in CCP2 markedly enhanced cofactor activity, while only moderately increasing the VCP-C $3 b$ affinity30. The equivalent residues in FH (Gln101, Ile106, Asp112 and Asp119) were fully exposed and found adjacent to conserved patches (Fig. 5a), which suggests a role for these four residues in the binding of FI. Furthermore, studies on cobra-venom factor (CVF) indicated that the $\mathrm{C} 345 \mathrm{C}$ domain of $\mathrm{C} 3 \mathrm{~b}$ may contribute to this interaction 31 . These data suggest that FI binds the C3b-FH complex at the area formed by CCP1-3 of FH and C345C and CUB of C3b (Supplementary Fig. 7 online). Besides providing a platform for FI, FH may have a putative second role in cofactor activity: we hypothesize that the bridge formed by CCP4 between TED and the core of C3b may maintain the position of TED while the connecting CUB domain undergoes further cleavage. This suggested function is supported by disease-related mutations in either FH CCP4 (refs. 32, 33) and in TED of C3b34, which indicates a functional importance for RCA interactions with TED.

\section{Mapping of disease-related mutations}

The complex structure provides a structural basis for understanding the effects of six disease-related mutations that can be found in domains CCP1-4 of FH. In a first step, we examined those three mutations (R60G, P204L, K2064) that are directly located at the C3bFH(1-4) interface (Fig. 3). In case of the aHUS-related mutant R60G35, a salt bridge between the guanidium group of Arg60 in FH CCP1 and the acidic Asp732 of a'NT in C3b was lost by the mutation (Supplementary Fig. 8a online). In FH CCP4, the backbone amide of Lys206 formed a hydrogen bond with the carboxylate of Glu1138 in TED, whereas the lysine side chain formed an intra-molecular salt bridge with the carboxylate of Glu213 (Supplementary Fig. 8b online). The MPGN-II-associated deletion of this residue is likely to severely alter the local structure in $\mathrm{CCP} 4$, which would explain the reduced binding to $\mathrm{C} 3 \mathrm{~b}$ and loss of regulatory functions of FH32. In the aHUS-related mutant P240L33, the cispeptide conformation of Pro240 is likely to be essential for binding of CCP4 into a pocket formed by both MG1 and TED of C3b (Supplementary Fig. 8c online) and its mutation may directly affect the C3b-FH interaction. The remaining three mutations (V44I, R35H and R109L) were not located at the C3b-FH interface (Supplementary Fig. 8d,e online). The V44I mutant is not only associated with AMD and MPGN-II36, 37 but was recently found to show a strong association with polypodial choroidal vasculopathy38. Val44 is part of the hydrophobic core of the CCP1 domain and may therefore affect domain stability. Indeed, NMR studies demonstrated an effect on thermal stability of V44I, whereas only subtle structural differences could be detected between wild-type and mutant proteins12. Arg35 of the aHUS-related mutation R35H33 was solvent-exposed and located on the bottom part of CCP1 opposite to the C3b-interaction surface (Supplementary Fig. 8d online). Though little structural rearrangements have been observed for the $\mathrm{R} 35 \mathrm{H}$ mutant 12 , its localization on the putative FI-interaction face of FH may affect the binding to FI. The mutation R109L has been found in two patients with MPGN-II39. As the hydrophobic portion of the arginine 
side chain was buried in the hydrophobic core of the CCP2 domain packing against the conserved Trp116 (Supplementary Fig. 8e online), this mutation possibly affects the folding or stability of CCP2.

Recently, a number of aHUS-linked mutations were also described for C334; they showed large effects on C3b-MCP and smaller effects on C3b-FH binding. Mutations R570Q or R570W, A1072V, Q1139K were all located at the C3b-FH interface (Fig. 3) and decreased the binding of FH by 10-50\%34. In case of R570Q and R570W, the replacement of Arg570 may lead to a loss of electrostatic interactions (at $4 \AA$ distance) with Glu98 of FH (see Supplementary Fig. $8 \mathrm{f}$ online). In the A1072V mutant, the bulkier side chain of valine may sterically hinder interactions with Tyr225 of FH (Supplementary Fig. 8g online) and reduce C3b-FH binding. Mutation Q1139K introduces a positive charge that possibly leads to a salt bridge between Lys1139 and the preceding Asp1138 (Supplementary Fig. 8h online) and likely modifies the interactions with FH. Other mutations in the TED domain, such as D1093N (Fig. 3), may not be connected with FH(1-4) but rather with the secondary C3b binding site at CCP19-20 (ref. 11).

\section{DISCUSSION}

Tight regulation of the complement response on host cells and in circulation is critical for providing selectivity against foreign cells and preventing complement-mediated tissue damage. Considering their essential role in immune response and the increasing number of diseases with known or suspected involvement of complement regulators, detailed knowledge about the underlying molecular processes is very much desired. Our data is of high importance, in this respect, as it yields the first structure of $\mathrm{C} 3 \mathrm{~b}$ in complex with a complement regulator. It not only provides an insight into the function of the most abundant regulator, $\mathrm{FH}$, but also allows us to develop more general models of complement regulation. In agreement with previous observations10, the N-terminal four out of $20 \mathrm{CCP}$ domains of FH were functionally sufficient and showed both decay acceleration and cofactor activity. Fragment $\mathrm{FH}(1-4)$ bound $\mathrm{C} 3 \mathrm{~b}$ in an extended configuration, which results in a remarkable long contact interface that covers the whole flank of $\mathrm{C} 3 \mathrm{~b}$. The interface involved all four Nterminal CCP domains of FH and multiple domains of C3b including a'NT, MG1, 2, 6 and 7 , CUB and TED. Despite this large interface, the binding affinity of the two molecules was rather low, with a $\mathrm{K}_{\mathrm{D}}$ of $11 \mu \mathrm{M}$. This result is in good agreement with a previous study that compared the binding affinities of several $\mathrm{FH}$ fragments to $\mathrm{C} 3 \mathrm{~b}$ and is in a similar range as FH(19-20) but much weaker than full-length FH11.

The structure of the $\mathrm{C} 3 \mathrm{~b}-\mathrm{FH}(1-4)$ complex provides a molecular basis for understanding the two regulatory mechanisms, decay-acceleration and cofactor activity, by which proteins of the RCA family protect host cells. Based on the structure comparison of $\mathrm{C} 3 \mathrm{~b}-\mathrm{FH}(1-4)$ and $\mathrm{C} 3 \mathrm{bBb} 21$, we conclude that decay acceleration is mainly mediated by the first two Nterminal CCP domains of FH, which bind the a'NT, MG2 and MG6-7 domains of C3b. These domains likely dislocate the $\mathrm{Bb}$ protease fragment by electrostatic repulsion and steric hindrance from the convertase. The conserved location of functional residues in the homologous domains CCP2-3 of DAF suggest that similar displacement effects also play a role in case of this regulator24. Nevertheless, DAF and FH may differ in their precise 
mechanisms of dislocating $\mathrm{Bb}$ from $\mathrm{C} 3 \mathrm{~b}$, as a direct interaction with $\mathrm{Bb}$ was only observed for DAF but not for FH25. We therefore hypothesize that DAF interacts with the $\beta \mathrm{F}-\alpha 7$ loop of the VWA domain in $\mathrm{Bb}$ and hence induces a low-affinity conformation of the MIDAS that mediates binding of $\mathrm{Bb}$ to the $\mathrm{C}$-terminus of $\mathrm{C} 3 \mathrm{~b}$. More structural data, such as $\mathrm{C} 3 \mathrm{~b}$ $\mathrm{DAF}$ and DAF-Bb complexes, are needed to understand the difference in the precise mechanisms of DAF and FH.

Our data further suggest that the cofactor activity of $\mathrm{FH}$ in the conversion of $\mathrm{C} 3 \mathrm{~b}$ to iC $3 \mathrm{~b}$ is based on two essential mechanisms. Firstly, FH provides a contact interface for the initial binding of the protease FI to the $\mathrm{C} 3 \mathrm{~b}$-regulator complex and brings it into close proximity of the CUB domain of C3b. The four N-terminal CCP domains of FH bind alongside the CUB domain of C3b. The structural arrangement suggests that FI may bind CCP1-3 of FH and $\mathrm{C} 345 \mathrm{C}$ of $\mathrm{C} 3 \mathrm{~b}$, which is supported by VCP mutagenesis data30 and CVF chimeras31. Though FI is reported to directly yet weakly bind to $\mathrm{C} 3 \mathrm{~b}$ in the absence of $\mathrm{FH}$, the binding affinity substantially increases after adding FH29. The newly formed extended binding site between $\mathrm{FH}$ and $\mathrm{C} 3 \mathrm{~b}$ is likely to largely improve FI-binding affinity and, as a consequence, the cleavage rate. Secondly, FH enables the sequential cleavage of C3b via stabilization of the TED-CUB arrangement with respect to the core of the C3b molecule. We show that domain CCP4 bridges the TED and MG1 domains of C3b and argue that these interactions will keep TED in place during the cleavage by FI. In contrast to FH, MCP does not bind to the a'NT region of C3b, which explains its lack of decay acceleration activity 17,18. The cofactor activity of MCP, on the other hand, may be mediated in a similar way as that of FH. Recent binding experiments of disease-related mutations indicate that $\mathrm{MCP}$ also binds $\mathrm{C} 3 \mathrm{~b}$ via MG6 and TED34. Several of the C3 mutants that showed significant reduction in C3bMCP binding have mutations such as R570Q, R570W, A1072V and Q1139K that are all at the $\mathrm{C} 3 \mathrm{~b}-\mathrm{FH}$ interface 34 . However, the exact binding arrangement is likely to be different between the two regulators, since the $\mathrm{C} 3 \mathrm{~b}$ binding site in domain CCP4 of MCP, as derived from mutagenesis studies40, does not coincide fully with the corresponding site in $\mathrm{FH}$ CCP4.

Factor $\mathrm{H}$ has experienced an increasing interest in biomedical sciences during the past decade as it became evident that mutations and polymorphisms of this regulator are strongly associated with diseases such as aHUS, AMD and MPGN-II33,34. In case of AMD, FH is even considered the most consistent genetic risk factor41. While some effects may be attributed to altered glycosaminoglycan binding or changes in the secondary $\mathrm{C} 3 \mathrm{~b}$ binding site on CCP19-20, a number of mutations are located in CCP1-4 and were therefore mapped on the $\mathrm{C} 3 \mathrm{~b}-\mathrm{FH}(1-4)$ structure. Our analysis indicates that disruption of specific contacts (such as salt bridges) or the induction of local conformational changes may significantly alter the $\mathrm{C} 3 \mathrm{~b}-\mathrm{FH}$ interface. The specific yet rather weak interaction between $\mathrm{C} 3 \mathrm{~b}$ and $\mathrm{FH}(1-$ 4) may be essential for a fast and selective complement regulation, but also makes FH prone to functional interference by even small modifications in its sequence. The described mutations are therefore likely to weaken the affinity, which may lead to a loss of regulatory activity and, finally, to apparent disease states.

In conclusion, our structural data provide an important framework for understanding the two key molecular mechanisms of host protection by RCA proteins, decay acceleration and 
cofactor activity. We also offer a base for explaining the functional consequences of individual mutations related to dysfunctional complement regulation and associated diseases. In this respect, this study may pave the way for developing therapeutics that are directed at modulating complement activation.

\section{METHODS}

\section{Protein expression and purification}

C3 was isolated from human plasma and C3b was generated by limited trypsin treatment of $\mathrm{C} 3$ as previously described19, 42 with some modifications as described in the

Supplementary Methods. The DNA fragment encoding human FH domains CCP1-4, residues 1-246, was amplified by PCR from the full-length $C F H$ clone and ligated into a pSecTag2 B vector (Invitrogen). Recombinant FH(1-4), which contains a C-myc tag and hexahistidine tag at its carboxyl terminus, was expressed in HEK293T cells with plasmids using polyethenimine (linear, $\sim 25 \mathrm{kDa}$; Polysciences Inc.) and incubated in HybridomaSFM (Gibco/Invitrogen) containing 1\% fetal bovine serum (HyClone). Medium was harvested four days after transfection and concentrated prior to protein purification. $\mathrm{FH}(1-4)$ was purified by metal-affinity chromatography (Ni-NTA Superflow; Qiagen Inc.,) and eluted with $200 \mathrm{mM}$ imidazole in PBS, pH 8.0. All protein-containing fractions were pooled and dialyzed against $10 \mathrm{mM}$ phosphate buffer, $\mathrm{pH}$ 7.4, and further purified by ion-exchange chromatography using a Resource Q column (GE Healthcare Bio-Sciences Corp.) with a linear gradient of $0-300 \mathrm{mM} \mathrm{NaCl}$ over 30 column volumes. Purified $\mathrm{FH}(1-4)$ was dialyzed against $10 \mathrm{mM}$ Tris, $50 \mathrm{mM} \mathrm{NaCl}, \mathrm{pH} 7.4$ and concentrated to $1.7 \mathrm{mg} / \mathrm{ml}$ before crystallization. The purity of $\mathrm{FH}(1-4)$ was verified using SDS-PAGE.

\section{Crystallization, data collection and structure determination}

Purified C3b (30 mg/ml) and FH(1-4) $(1.7 \mathrm{mg} / \mathrm{ml})$ were mixed at a molar ratio of $1: 1$ to a final concentration $8.5 \mathrm{mg} / \mathrm{ml}(40 \mu \mathrm{M})$ of the complex. C3b-FH(1-4) was crystallized in $7.0 \%$ (w/v) PEG 3,350, $70 \mathrm{mM}$ ammonium acetate, $\mathrm{pH} 7.1$ by hanging-drop vapour diffusion at $18{ }^{\circ} \mathrm{C}$. Crystals appeared after 2 days and grew within 5 days to a typical size of $200 \times 100 \times 60 \mu \mathrm{m}$. The crystals were soaked in reservoir solution supplemented with $20 \%$ glycerol for several minutes and then flash-cooled in liquid nitrogen. The crystals belong to space group $P 2{ }_{1} 2{ }_{1} 2(a=223.4, b=84.9, c=128.8 \AA)$ and the best crystal diffracted to 2.7A resolution at ESRF beamline ID14-EH4. Diffraction data was integrated and scaled with MOSFLM and SCALA43. The structure of C3b-FH(1-4) was determined by molecular replacement using PHASER44. First, the $\beta$-chain (residues $1-642$ ) of C3c (pdb code 2A74) 19 was placed, followed by the stepwise addition of TED, CUB and a'NT-MG7-MG8 domains from C3b (pdb code: 2I07)14. The initial models for the individual CCP domains of FH were generated by the program Chainsaw 45 using structures of human $\beta 2$ glycoprotein I CCP2 (pdb code: 1QUB, residues 63-121)46 and DAF CCP4 (pdb code: 1H03, residues 130-191)23 as templates. CCP2 and CCP3 domains of FH were found, but molecular replacement searches of domains CCP1 and CCP4 initially failed. The C345C domain of $\mathrm{C} 3 \mathrm{~b}$ was then successfully placed using the $\mathrm{C} 345 \mathrm{C}$ domain from the $\mathrm{C} 3 \mathrm{c}$ structure (residues 1496-1641) as search model. The chainsaw model for the fourth CCP domain of FH was placed manually using COOT47, based on a $F o-F c$ difference map and 
subsequently refined as a rigid body in PHASER. After several rounds of refinement and model building in COOT, the bottom part of CCP1 (20 of 65 residues in total) could be built manually. The complete CCP1 derived from the NMR structure of FH CCP1-2 (pdb code: 2RLP, residues 2-64)12 was then placed by superposition and rigid-body refinement in PHASER to obtain the complete model. The model was refined using REFMAC48 and PHENIX49. The final model consisting of 1,536 residues of C3b and 244 residues of $\mathrm{FH}(1-$ 4), had $R_{\text {work }}$ and $R_{\text {free }}$ values of 21.7 and $25.2 \%$, respectively.

\section{Binding affinity assay}

Surface plasmon resonance (SPR) was used to determine the binding affinity of soluble FH(1-4) for thioester-specifically immobilized C3b as described in the Supplementary Methods.

\section{Complement decay acceleration and cofactor activity}

The decay acceleration activity assay for the alternative pathway of complement activation was performed by SPR. On-chip formation and decay of the C3 convertase was observed by injecting an equimolar mixture of factor $\mathrm{B}$ and factor $\mathrm{D}(100 \mathrm{nM}$ each) on immobilized $\mathrm{C} 3 \mathrm{~b}$ (see Supplementary Methods) for $2 \mathrm{~min}$ at a flow rate of $10 \mu \mathrm{l} / \mathrm{min}$ in HBS-Mg ${ }^{2+}$ buffer (10 mM HEPES, $150 \mathrm{mM} \mathrm{NaCl}, 0.005 \%$ Tween-20, $1 \mathrm{mM} \mathrm{MgCl}_{2}$, pH 7.4). After an undisturbed decay phase of $3 \mathrm{~min}, 200 \mathrm{nM}$ of either $\mathrm{FH}(1-20)$, FH(1-4), FH(19-20) or a buffer control was injected for $1 \mathrm{~min}$ and the drop in post-injection baseline was evaluated. To remove residual interactants, the surface was regenerated using $1 \mu \mathrm{M} \mathrm{FH}(1-4)$ and $1 \mathrm{M}$ $\mathrm{NaCl}$. The binding signals of the $\mathrm{FH}$ fragments with $\mathrm{C} 3 \mathrm{~b}$ (in absence of the convertase) have been subtracted from the data set in order to visualize the pure decay acceleration. The cofactor activity of $\mathrm{FH}(1-4)$ was tested using a previously described protocol50. Briefly, 4 $\mu \mathrm{g}$ of human $\mathrm{C} 3 \mathrm{~b}(1.14 \mu \mathrm{M})$ and $10 \mathrm{ng}$ of human factor $\mathrm{I}(8 \mathrm{nM})$ were incubated with various amounts $(9,18,36 \mathrm{nM})$ of $\mathrm{FH}(1-20)$ or $\mathrm{FH}(1-4)$ at $37^{\circ} \mathrm{C}$ for $1 \mathrm{~h}$ in PBS buffer at a final volume of $20 \mu \mathrm{l}$. The reaction was stopped by adding SDS-PAGE loading buffer and the samples were analyzed with $10 \%$ SDS-PAGE.

\section{Antibody competition assay}

Individual contact sites of $\mathrm{FH}(1-4)$ on $\mathrm{C} 3 \mathrm{~b}$ were confirmed by SPR using monoclonal anti$\mathrm{C} 3 \mathrm{~b}$ antibodies as described in the Supplementary Methods.

\section{Supplementary Material}

Refer to Web version on PubMed Central for supplementary material.

\section{Acknowledgements}

We gratefully thank the European Synchrotron Radiation Facility (ESRF) for the provision of synchrotron radiation facilities; and beamline scientists of the ESRF and the European Molecular Biology Laboratory for assistance. We thank M. Pangburn (University of Texas, Tyler TX, USA) for providing the $C F H$ clone and P. Barlow (University of Edinburgh, UK) for providing the FH(19-20) protein. We thank M.A. Hadders for critically reading the manuscript and helpful comments. This work was supported by a 'Top' grant (to P.G.) by the Council for Chemical Sciences of the Netherlands Organization for Scientific Research (NWO-CW) and NIH-grants (to J.D.L.). 


\section{REFERENCES}

1. Schmidt CQ, Herbert AP, Hocking HG, Uhrin D, Barlow PN. Translational Mini-Review Series on Complement Factor H: Structural and functional correlations for factor H. Clinical \& Experimental Immunology. 2008; 151:14-24. [PubMed: 18081691]

2. Liszewski MK, Farries TC, Lublin DM, Rooney IA, Atkinson JP. Control of the complement system. Adv Immunol. 1996; 61:201-283. [PubMed: 8834497]

3. Prosser BE, et al. Structural basis for complement factor $\mathrm{H}$ linked age-related macular degeneration. J. Exp. Med. 2007; 204:2277-2283. [PubMed: 17893204]

4. Meri S. Loss of self-control in the complement system and innate autoreactivity. Ann N Y Acad Sci. 2007; 1109:93-105. [PubMed: 17785294]

5. de Cordoba SR, de Jorge EG. Translational mini-review series on complement factor H: genetics and disease associations of human complement factor H. Clin Exp Immunol. 2008; 151:1-13. [PubMed: 18081690]

6. Lambris JD, Ricklin D, Geisbrecht BV. Complement evasion by human pathogens. Nat Rev Microbiol. 2008; 6:132-142. [PubMed: 18197169]

7. Schneider MC, et al. Neisseria meningitidis recruits factor $\mathrm{H}$ using protein mimicry of host carbohydrates. Nature. 2009; 458:890-893. [PubMed: 19225461]

8. Ricklin D, Lambris JD. Complement-targeted therapeutics. Nat Biotechnol. 2007; 25:1265-1275. [PubMed: 17989689]

9. Noris M, Remuzzi G. Translational Mini-Review Series on Complement Factor H: Therapies of renal diseases associated with complement factor $\mathrm{H}$ abnormalities: atypical haemolytic uraemic syndrome and membranoproliferative glomerulonephritis. Clinical \& Experimental Immunology. 2008; 151:199-209. [PubMed: 18070148]

10. Gordon DL, Kaufman RM, Blackmore TK, Kwong J, Lublin DM. Identification of complement regulatory domains in human factor H. J Immunol. 1995; 155:348-356. [PubMed: 7541419]

11. Schmidt CQ, et al. A new map of glycosaminoglycan and C3b binding sites on factor $\mathrm{H}$. $\mathrm{J}$ Immunol. 2008; 181:2610-2619. [PubMed: 18684951]

12. Hocking HG, et al. Structure of the $\mathrm{N}$-terminal region of complement factor $\mathrm{H}$ and conformational implications of disease-linked sequence variations. J Biol Chem. 2008; 283:9475-9487. [PubMed: 18252712]

13. Okemefuna AI, et al. The regulatory SCR-1/5 and cell surface-binding SCR-16/20 fragments of factor $\mathrm{H}$ reveal partially folded-back solution structures and different self-associative properties. $\mathrm{J}$ Mol Biol. 2008; 375:80-101. [PubMed: 18005991]

14. Janssen BJ, Christodoulidou A, McCarthy A, Lambris JD, Gros P. Structure of C3b reveals conformational changes that underlie complement activity. Nature. 2006; 444:213-216. [PubMed: 17051160]

15. Wiesmann C, et al. Structure of C3b in complex with CRIg gives insights into regulation of complement activation. Nature. 2006; 444:217-220. [PubMed: 17051150]

16. Becherer JD, Alsenz J, Esparza I, Hack CE, Lambris JD. Segment spanning residues 727-768 of the complement $\mathrm{C} 3$ sequence contains a neoantigenic site and accommodates the binding of CR1, factor H, and factor B. Biochemistry. 1992; 31:1787-1794. [PubMed: 1371073]

17. Lambris JD, et al. Dissection of CR1, factor $\mathrm{H}$, membrane cofactor protein, and factor $\mathrm{B}$ binding and functional sites in the third complement component. J Immunol. 1996; 156:4821-4832. [PubMed: 8648130]

18. Oran AE, Isenman DE. Identification of residues within the 727-767 segment of human complement component $\mathrm{C} 3$ important for its interaction with factor $\mathrm{H}$ and with complement receptor 1 (CR1, CD35). J Biol Chem. 1999; 274:5120-5130. [PubMed: 9988761]

19. Janssen BJ, et al. Structures of complement component $C 3$ provide insights into the function and evolution of immunity. Nature. 2005; 437:505-511. [PubMed: 16177781]

20. Gros P, Milder FJ, Janssen BJ. Complement driven by conformational changes. Nat Rev Immunol. 2008; 8:48-58. [PubMed: 18064050] 
21. Rooijakkers SHM, et al. Structure of the complement convertase stabilized by a staphylococcal inhibitor. to be published. 2009

22. Hourcade DE, Mitchell L, Kuttner-Kondo LA, Atkinson JP, Medof ME. Decay-accelerating factor (DAF), complement receptor 1 (CR1), and factor $\mathrm{H}$ dissociate the complement AP C3 convertase $(\mathrm{C} 3 \mathrm{bBb})$ via sites on the type A domain of Bb. J Biol Chem. 2002; 277:1107-1112. [PubMed: 11694537]

23. Lukacik P, et al. Complement regulation at the molecular level: the structure of decay-accelerating factor. Proc Natl Acad Sci U S A. 2004; 101:1279-1284. [PubMed: 14734808]

24. Kuttner-Kondo L, et al. Structure-based mapping of DAF active site residues that accelerate the decay of C3 convertases. J Biol Chem. 2007; 282:18552-18562. [PubMed: 17395591]

25. Harris CL, Abbott RJ, Smith RA, Morgan BP, Lea SM. Molecular dissection of interactions between components of the alternative pathway of complement and decay accelerating factor (CD55). J Biol Chem. 2005; 280:2569-2578. [PubMed: 15536079]

26. Luo BH, Carman CV, Springer TA. Structural basis of integrin regulation and signaling. Annu Rev Immunol. 2007; 25:619-647. [PubMed: 17201681]

27. Goicoechea de Jorge E, et al. Gain-of-function mutations in complement factor B are associated with atypical hemolytic uremic syndrome. Proc Natl Acad Sci U S A. 2007; 104:240-245. [PubMed: 17182750]

28. Sahu A, Isaacs SN, Soulika AM, Lambris JD. Interaction of vaccinia virus complement control protein with human complement proteins: factor I-mediated degradation of $\mathrm{C} 3 \mathrm{~b}$ to iC $3 \mathrm{~b} 1$ inactivates the alternative complement pathway. J Immunol. 1998; 160:5596-5604. [PubMed: 9605165]

29. DiScipio RG. Ultrastructures and interactions of complement factors H and I. J Immunol. 1992; 149:2592-2599. [PubMed: 1401896]

30. Yadav VN, Pyaram K, Mullick J, Sahu A. Identification of hot spots in the variola virus complement inhibitor (SPICE) for human complement regulation. J Virol. 2008; 82:3283-3294. [PubMed: 18216095]

31. Fritzinger DC, et al. Functional characterization of human $\mathrm{C} 3 /$ cobra venom factor hybrid proteins for therapeutic complement depletion. Developmental \& Comparative Immunology. 2009; 33:105-116. [PubMed: 18760301]

32. Licht $\mathrm{C}$, et al. Deletion of Lys224 in regulatory domain 4 of Factor $\mathrm{H}$ reveals a novel pathomechanism for dense deposit disease (MPGN II). Kidney Int. 2006; 70:42-50. [PubMed: 16612335]

33. Saunders RE, Goodship TH, Zipfel PF, Perkins SJ. An interactive web database of factor Hassociated hemolytic uremic syndrome mutations: insights into the structural consequences of disease-associated mutations. Hum Mutat. 2006; 27:21-30. [PubMed: 16281287]

34. Fremeaux-Bacchi V, et al. Mutations in complement $\mathrm{C} 3$ predispose to development of atypical hemolytic uremic syndrome. Blood. 2008; 112:4948-4952. [PubMed: 18796626]

35. Caprioli J, et al. Complement factor $\mathrm{H}$ mutations and gene polymorphisms in haemolytic uraemic syndrome: the C-257T, the A2089G and the G2881T polymorphisms are strongly associated with the disease. Hum Mol Genet. 2003; 12:3385-3395. [PubMed: 14583443]

36. Hageman GS, et al. A common haplotype in the complement regulatory gene factor $\mathrm{H}(\mathrm{HF} 1 / \mathrm{CFH})$ predisposes individuals to age-related macular degeneration. Proc Natl Acad Sci U S A. 2005; 102:7227-7232. [PubMed: 15870199]

37. Abrera-Abeleda MA, et al. Variations in the complement regulatory genes factor $\mathrm{H}(\mathrm{CFH})$ and factor $\mathrm{H}$ related 5 (CFHR5) are associated with membranoproliferative glomerulonephritis type II (dense deposit disease). J Med Genet. 2006; 43:582-589. [PubMed: 16299065]

38. Kondo N, Honda S, Kuno S, Negi A. Coding variant I62V in the complement factor $\mathrm{H}$ gene is strongly associated with polypoidal choroidal vasculopathy. Ophthalmology. 2009; 116:304-310. [PubMed: 19187823]

39. Dragon-Durey MA, et al. Heterozygous and homozygous factor $\mathrm{h}$ deficiencies associated with hemolytic uremic syndrome or membranoproliferative glomerulonephritis: report and genetic analysis of 16 cases. J Am Soc Nephrol. 2004; 15:787-795. [PubMed: 14978182] 
40. Liszewski MK, et al. Dissecting sites important for complement regulatory activity in membrane cofactor protein (MCP; CD46). J Biol Chem. 2000; 275:37692-37701. [PubMed: 10960475]

41. Coleman HR, Chan CC, Ferris FL, Chew EY 3rd. Age-related macular degeneration. Lancet. 2008; 372:1835-1845. [PubMed: 19027484]

42. Lambris JD, Dobson NJ, Ross GD. Release of endogenous C3b inactivator from lymphocytes in response to triggering membrane receptors for beta $1 \mathrm{H}$ globulin. J. Exp. Med. 1980; 152:16251644. [PubMed: 6450259]

43. Evans P. Scaling and assessment of data quality. Acta Crystallographica Section D. 2006; 62:7282.

44. McCoy AJ, et al. Phaser crystallographic software. Journal of Applied Crystallography. 2007; 40:658-674. [PubMed: 19461840]

45. Stein N. CHAINSAW: a program for mutating pdb files used as templates in molecular replacement. Journal of Applied Crystallography. 2008; 41:641-643.

46. Bouma B, et al. Adhesion mechanism of human beta(2)-glycoprotein I to phospholipids based on its crystal structure. EMBO J. 1999; 18:5166-5174. [PubMed: 10508150]

47. Emsley P, Cowtan K. Coot: model-building tools for molecular graphics. Acta Crystallogr D Biol Crystallogr. 2004; 60:2126-2132. [PubMed: 15572765]

48. Winn MD, Murshudov GN, Papiz MZ. Macromolecular TLS refinement in REFMAC at moderate resolutions. Methods Enzymol. 2003; 374:300-321. [PubMed: 14696379]

49. Adams PD, et al. PHENIX: building new software for automated crystallographic structure determination. Acta Crystallogr D Biol Crystallogr. 2002; 58:1948-1954. [PubMed: 12393927]

50. Alsenz J, Lambris JD, Schulz TF, Dierich MP. Localization of the complement-component-C3bbinding site and the cofactor activity for factor $\mathrm{I}$ in the $38 \mathrm{kDa}$ tryptic fragment of factor $\mathrm{H}$. Biochem J. 1984; 224:389-398. [PubMed: 6240261] 
a
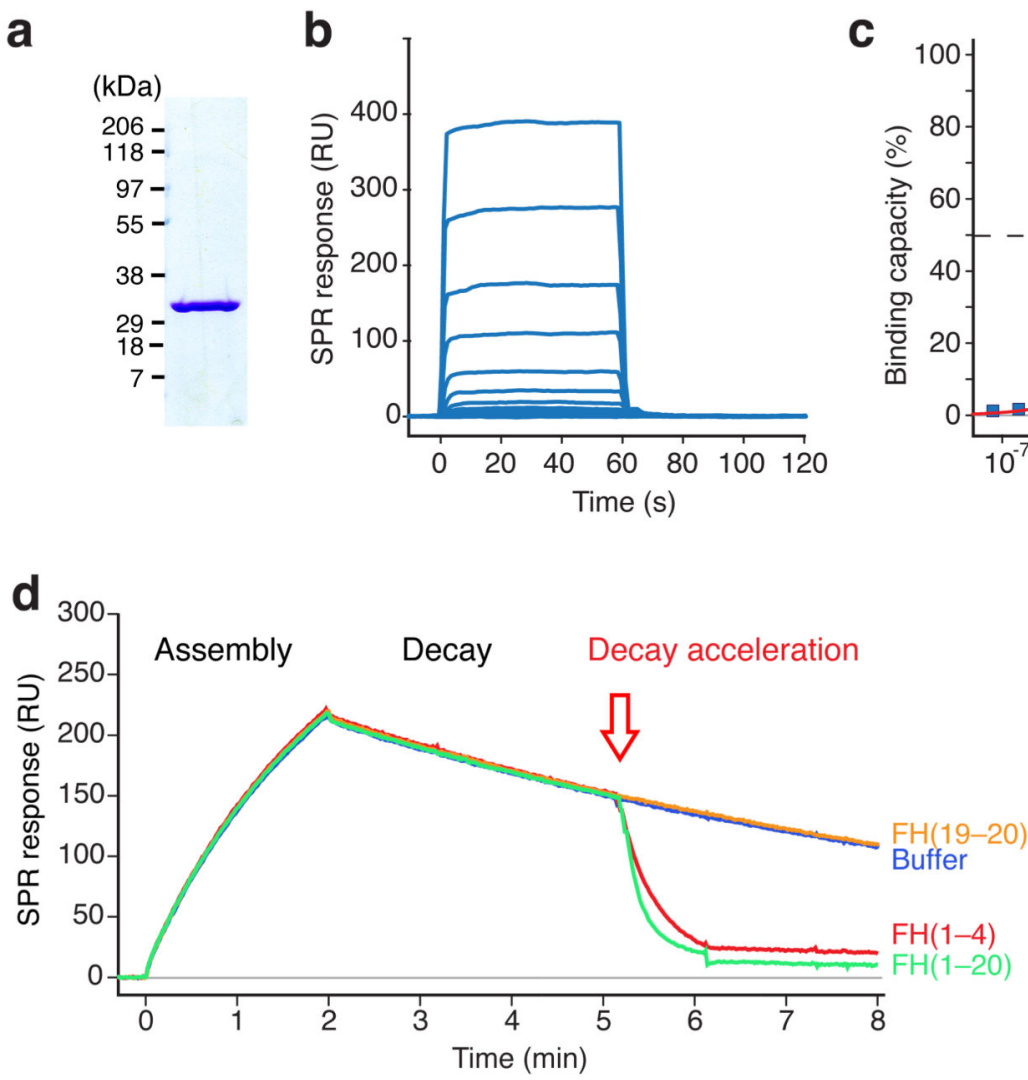

C

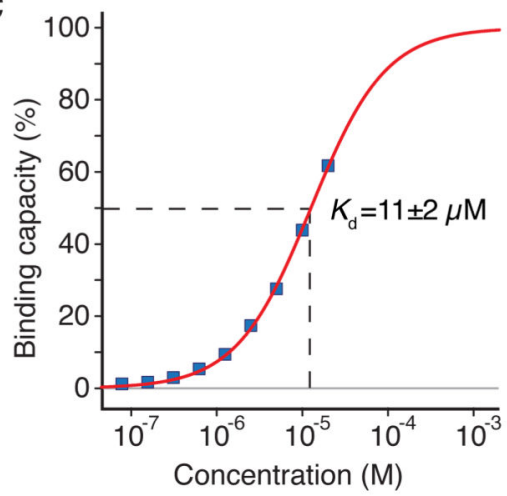

e

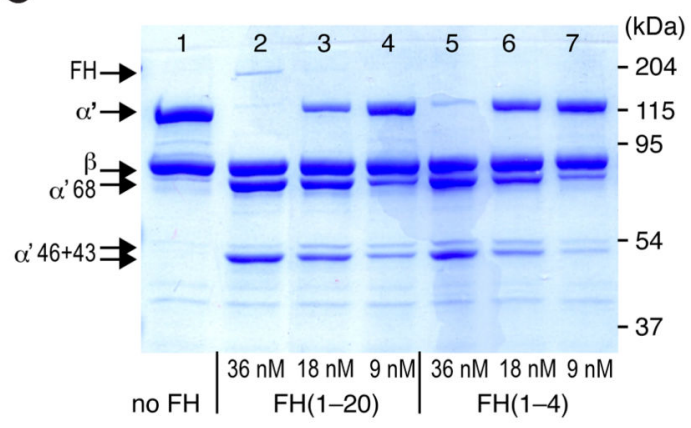

Figure 1.

Characterization of recombinant $\mathrm{FH}(1-4)$. (a) Purity of FH(1-4) as assessed by SDS-PAGE (4-15\% gradient gel; reducing conditions; Coomassie blue staining). (b-c) Direct binding between soluble $\mathrm{FH}(1-4)$ and surface-bound $\mathrm{C} 3 \mathrm{~b}$. Injection of the FH fragment (0.08-20 $\mu \mathrm{M}$ ) leads to the formation of a short-lived 1:1 complex with $\mathrm{C} 3 \mathrm{~b}$, which features a binding affinity of $K_{D}=11 \pm 2 \mu \mathrm{M}$. Data are representative of five experiments with different surface densities of C3b (3,000-7,000 RU; resonance units). (d) FH(1-4) features a comparable decay acceleration activity as full-length FH when injected onto surface-based $\mathrm{C} 3$ convertase $(\mathrm{C} 3 \mathrm{bBb})$. Additional details and control injections for the decay acceleration assay can be found in Supplementary Fig. 9 online. Data are representative of three independent experiments. (e) The cofactor activity of $\mathrm{FH}(1-4)$ is similar to that of $\mathrm{FH}$ : incubation of $\mathrm{C} 3 \mathrm{~b}$ with FI and increasing amounts of either $\mathrm{FH}(1-4)$ or $\mathrm{FH}(1-20)$ leads to the generation of $\mathrm{iC} 3 \mathrm{~b}$ as visible by the degradation of the a' chain to three fragments of 43 , 46 , and $68 \mathrm{kDa}$, respectively. Data are representative of more than five individual experiments. 


\section{b}
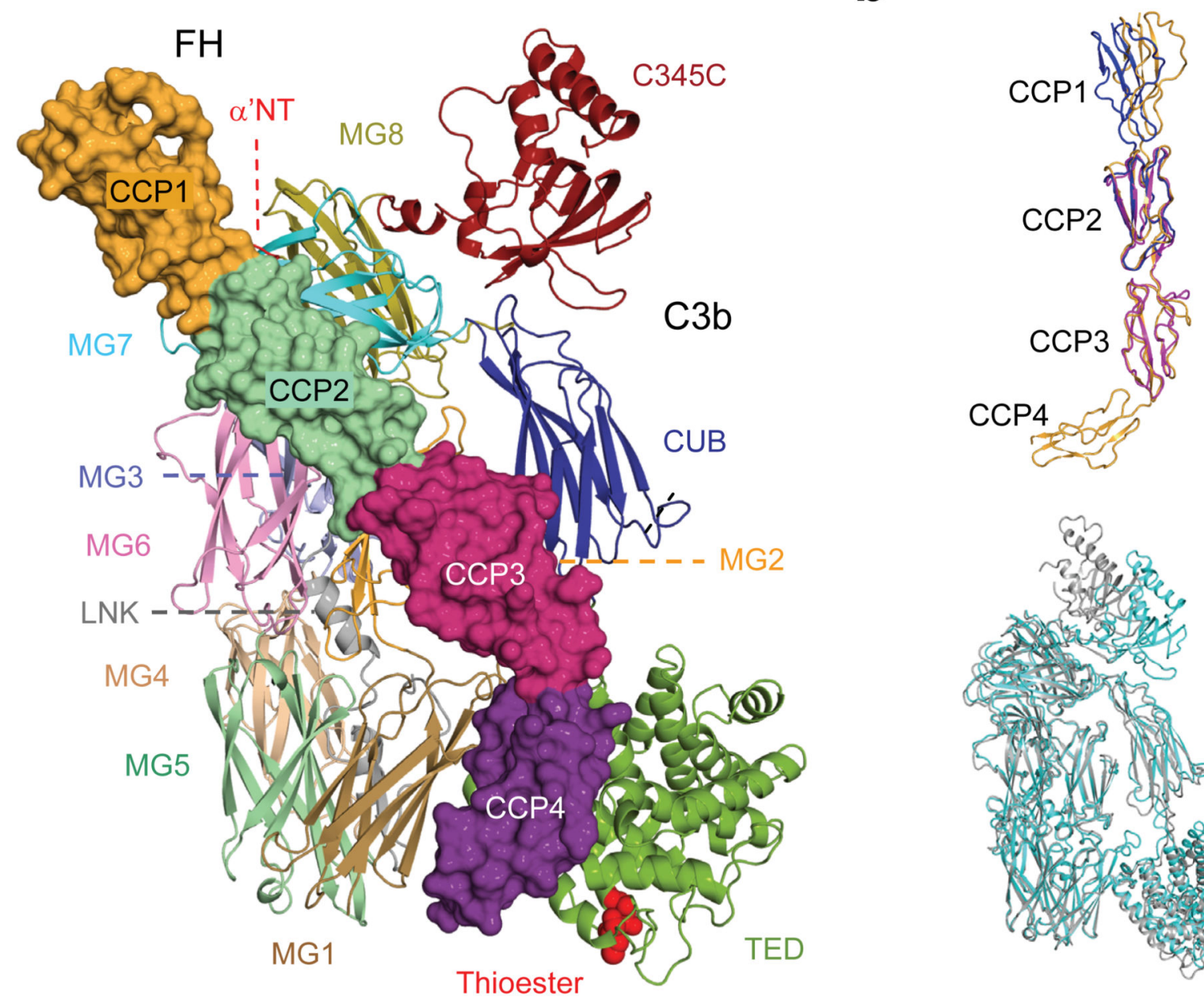

Figure 2.

Structure of $\mathrm{C} 3 \mathrm{~b}$ in complex with FH domains CCP1-4. (a) Overall structure of the C3b$\mathrm{FH}(1-4)$ complex. $\mathrm{C} 3 \mathrm{~b}$ is shown in ribbon and $\mathrm{FH}$ is shown in surface representations; domains are indicated and the thioester is shown by red spheres. (b) On the left-hand side, comparison of the FH CCP1-4 as observed in the complex (orange) with solution structures of CCP1-2 (blue) and CCP2-3 (magenta)12; and, on the right-hand side, comparison of C3b as observed in complex with $\mathrm{FH}(1-4)$ (cyan) and free C3b (grey)14. Figures were generated by using PyMOL (http://www.pymol.org). 

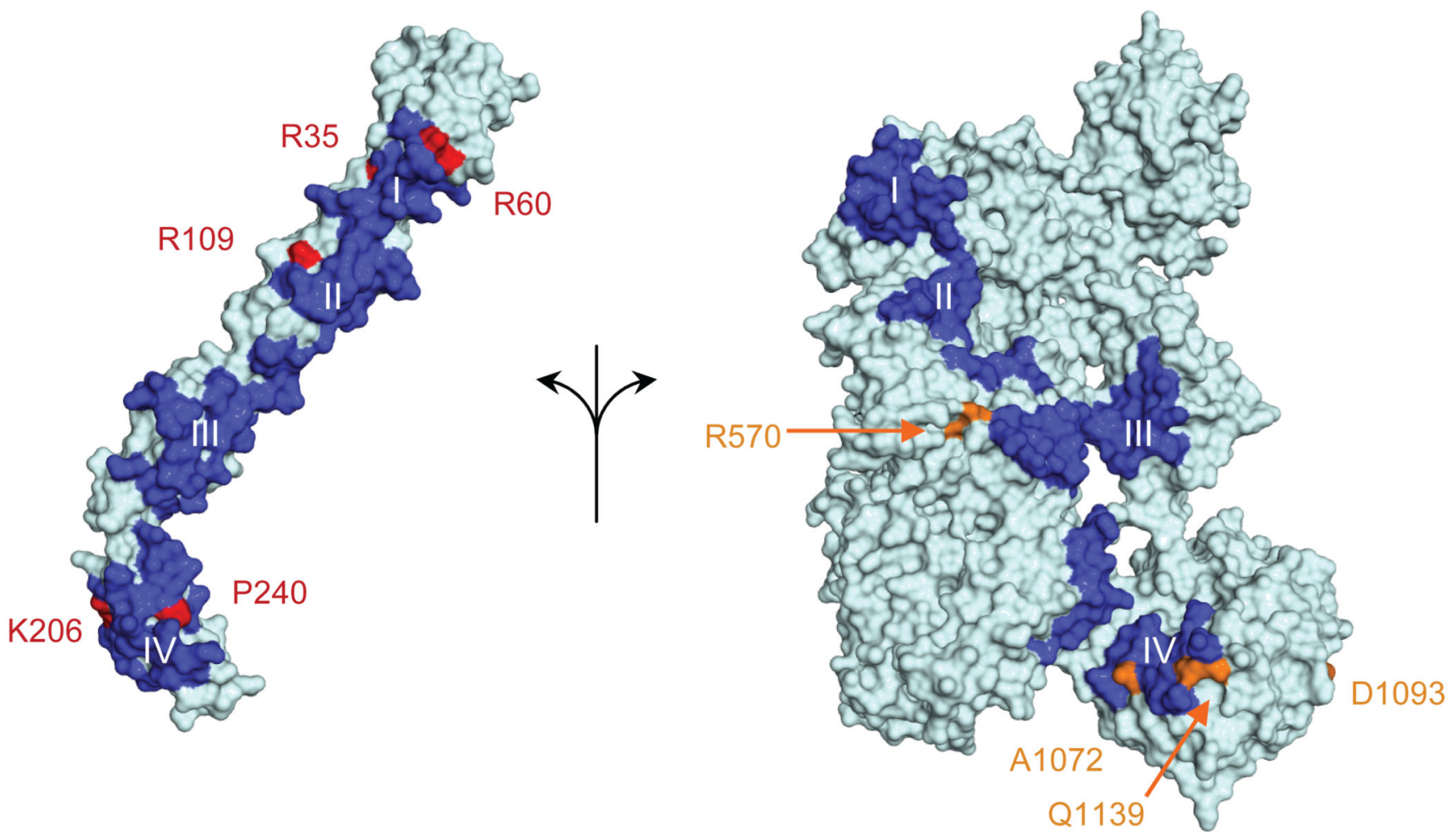

Figure 3.

Mapping FH and C3 mutants on complex structure. The molecules are shown in surface representation with $\mathrm{FH}(1-4)$ on the left side and $\mathrm{C} 3 \mathrm{~b}$ on the right side. The four contact regions (I-IV) between $\mathrm{FH}(1-4)$ and $\mathrm{C} 3 \mathrm{~b}$ are highlighted in blue with mutations related to AMD, MPGN-II and aHUS are indicated in red (for FH mutants) and orange (for C3b mutants). 
a
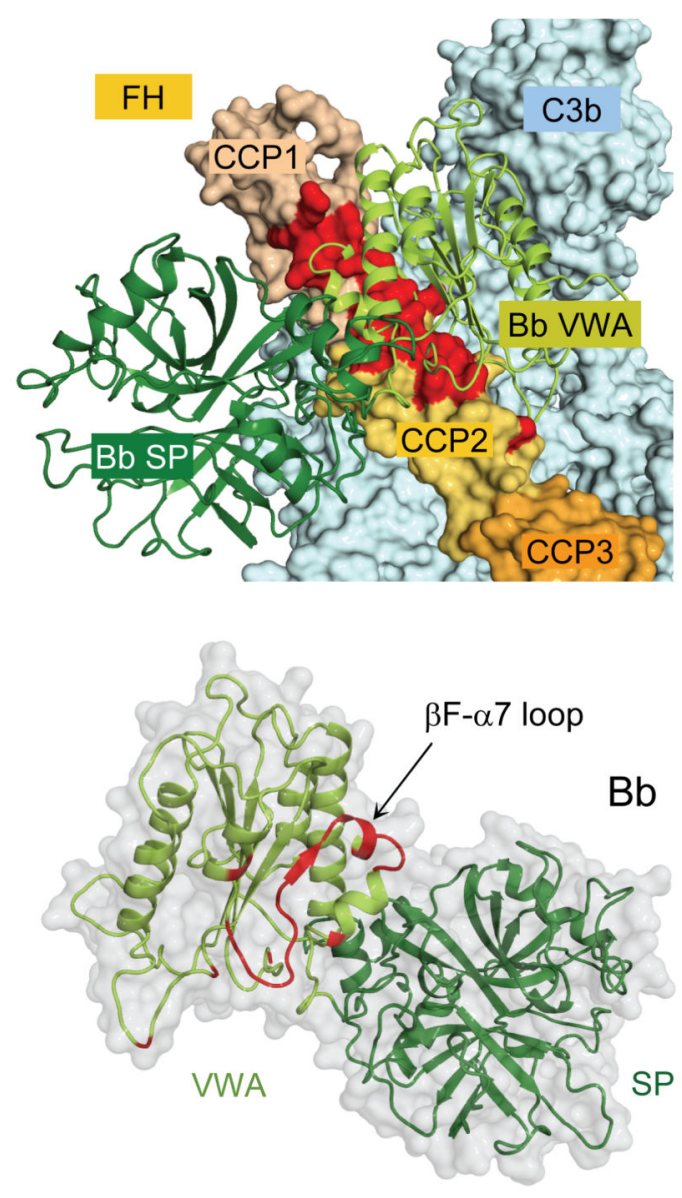

b
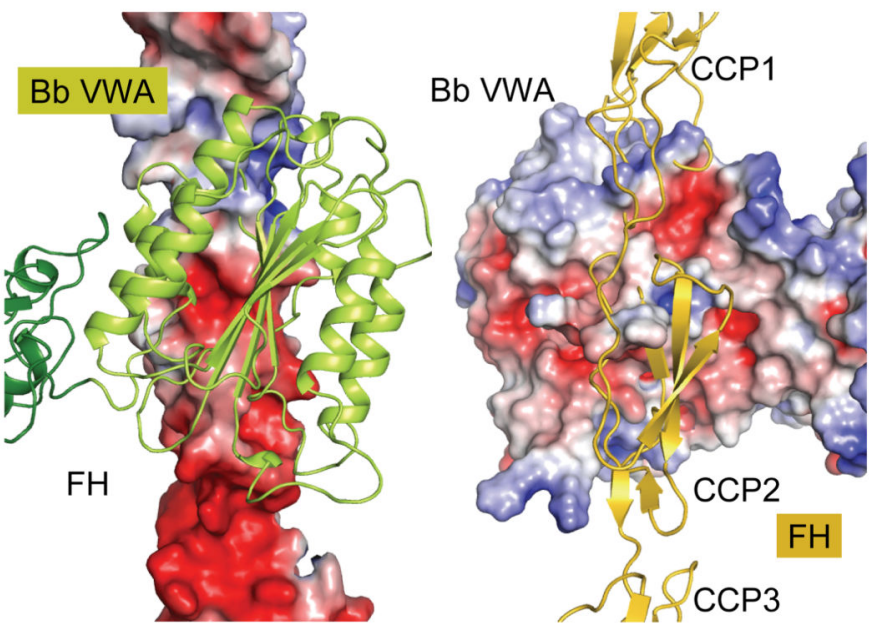

C

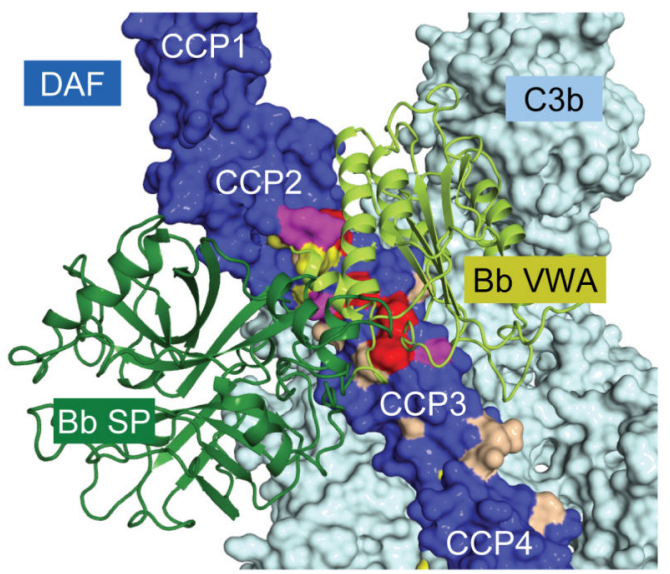

Figure 4.

Structural basis of decay acceleration activity. (a) Overlay of the C3b-FH and C3b-Bb complexes21 (top figure) with C3b (light cyan) and FH CCP1-3 (beige, yellow orange and orange, respectively) in surface representation and $\mathrm{Bb}$ in ribbon representation (light green and dark green for VWA and SP domains, respectively); the red surface area indicates the region where $\mathrm{FH}$ and $\mathrm{Bb}$ overlap (atomic distances less than $2 \AA$ ). Bottom figure shows the overlap region (red) in $\mathrm{Bb}\left(\mathrm{Bb}\right.$ is rotated by $180^{\circ}$ with respect to the top figure). (b) Electrostatic surface potential of $\mathrm{FH}$ and ribbon representation of $\mathrm{Bb}$ (left side) and vice versa (right side) in the regions facing each other. The potential contours are shown on a scale from -5 (red) to $+5 k_{b} T_{e_{c}}{ }^{-1}$ (blue). (c) Superposition of DAF CCP2-423 on to FH CCP1-3 shown in the same representation as in panel a. Mutants of DAF are coloured by degree of functional interference, from minor (beige), medium (yellow) and severe (magenta) effect up to complete abortion (red) of decay acceleration by DAF24. 
a

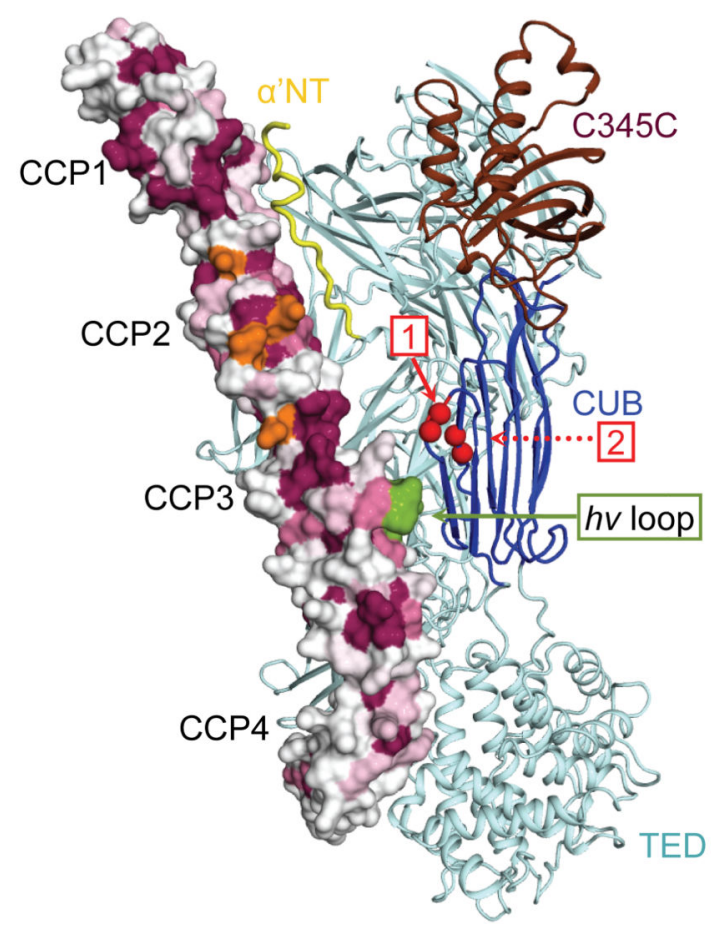

b

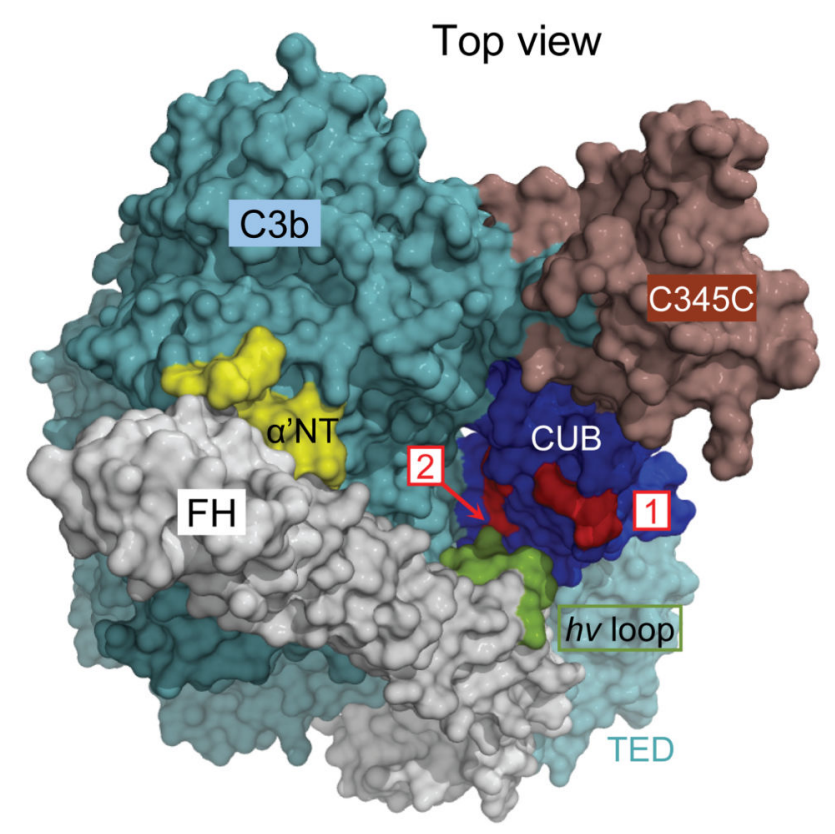

Figure 5.

Structural implications for cofactor activity. (a) Sites of FI binding and cleavage. FH is shown in surface representation and colour-coded to residue conservation with mutational data from ref. 30. The conservation scale and corresponding colours are indicated in Supplementary Fig. 4 online. Residues with the conservation scores less than 5 are all coloured in white. Mutations in VCP enhancing FI binding are indicated in orange and the hyper-variable loop ( $h v$-loop) of CCP3 in green. C3b (cyan) is shown in cartoon representation with the CUB (blue), a'NT (yellow) and C345C (dark red) domains highlighted and the first and second scissile bond in the CUB domain indicated by red spheres. (b) Surface representation of the complex from the top view. Domains of C3b are coloured the same as in panel a, and FH domains are in grey. The colouring of domains and FI cleavage sites is consistent with $\mathbf{a}$. 


\section{Table 1}

\section{Data collection and refinement statistics}

\begin{tabular}{ll}
\hline & $\mathbf{2 . 7 - \AA}$ \\
\hline Data collection & \\
Space group & $P 2{ }_{1} 22$ \\
Cell dimensions & \\
$\quad$ a, $b, c(\AA)$ & $223.5,84.9,128.8$ \\
Resolution $(\AA)$ & $67-2.7(2.85-2.7)^{*}$ \\
$R_{\text {merge }}(\%)$ & $11.3 \%(60.8)$ \\
$I / \sigma I$ & $8.3(1.9)$ \\
Completeness $(\%)$ & $99.7(100)$ \\
Redundancy & $3.7(3.8)$ \\
Refinement & \\
Resolution $(\AA)$ & $64.5-2.7$ \\
No. reflections & 67893 \\
$R$ work/ $R_{\text {free }}(\%)$ & $21.7 / 25.2$ \\
No. atoms & 14442 \\
Protein & 14081 \\
Ligand/ion & 189 \\
Water & 172 \\
B-factors $\left(\AA^{2}\right)$ & \\
Protein & 68.2 \\
Ligand/ion & 106.7 \\
Water & 48.2 \\
R.m.s deviations & \\
Bond lengths $(\AA)$ & 0.002 \\
Bond angles $\left({ }^{\circ}\right)$ & 0.446 \\
\hline & \\
&
\end{tabular}

*Highest resolution shell is shown in parenthesis. 